\title{
Viewpoint alignment and response conflict during spatial judgment
}

\author{
MYEONG-HO SOHN \\ Carnegie Mellon University, Pittsburgh, Pennsylvania \\ and \\ RICHARD A. CARLSON \\ Pennsylvania State University, University Park, Pennsylvania
}

\begin{abstract}
Spatial judgment (e.g., identifying the relative location, such as left or right, of a target) from a reference point becomes more difficult with increasing disparity between the relevant allocentric viewpoint and the observer-centered viewpoint. The viewpoint alignment hypothesis suggests that this misalignment effect is due to a realignment process that reconciles two viewpoints, implying that providing an advance cue for a viewpoint should facilitate the subsequent judgment. We examined whether advance viewpoint information can reduce the misalignment effect and whether the misalignment effect reflects response conflict, as well as realignment of viewpoints. In Experiment 1, the misalignmenteffect decreased with advance viewpoint information, suggesting that the misalignment effect indeed reflects viewpoint realignment. In Experiment 2, the misalignment effect was greater with spatial response codes that might conflict with the spatially arranged response keys than with arbitrary responses with no such conflict. These results suggest that the misalignment effect may arise from both viewpoint realignment and response conflicts.
\end{abstract}

Despite the enormous complexity of the perceptual, cognitive, and motor processes involved in spatial cognition, it often seems almost trivial to navigate through the environment successfully. This is possible because one's own viewpoint is always available to the navigating self. However, the constant availability of the observer-centered viewpoint is also presumably the most constraining factor in spatial cognition. Spatial cognition tasks often require adopting an allocentric viewpoint, one that is centered on an external object, a location, or another human being (Klatzky, 1998), and it can be very challenging to comprehend the environment from a viewpoint other than one's own. Spatial cognition studies suggest that this difficulty may be due to conflict between the observer-centered and the allocentric viewpoints. For example, children's difficulty describing a scene from someone else's perspective is often associated with errors reflecting their own viewpoints (Liben \& Downs, 1993; Rieser, Garing, \& Young, 1994). Adults are not much different. In map-reading studies, performance on pointing or reaching tasks is slower and less accurate when the orien-

Parts of this research were presented in the 37th Annual Meeting of the Psychonomic Society, Chicago. We thank Veronica Dark, Craig Haimson, and Beverly Roskos-Ewoldsen for their helpful comments on earlier drafts. Correspondence concerning this article should be addressed to M.-H. Sohn, BH 345E, Department of Psychology, Carnegie Mellon University, Pittsburgh, PA 15213 (e-mail: mhsohn+ @ andrew.cmu. edu).

Note-This article was accepted by the previous editorial team, while John T. Wixted was editor. tation of a map is misaligned with a participant's viewpoint (Levine, Jankovic, \& Palij, 1982; Presson \& Hazelrigg, 1984). Target detection is slower when a target is spatially defined from an allocentric viewpoint than when defined from the observer-centered viewpoint (Logan, 1995). When a familiar object is presented in a noncanonical orientation, people take longer to recognize the object (Cooper \& Shepard, 1973; Hinton \& Parsons, 1981). These results demonstrate the misalignment effect, the increase in spatial judgment errors or latencies as the relevant allocentric reference frame is misaligned with the observer-centered reference frame.

The misalignment effect might reflect the cost of adopting an allocentric viewpoint and its associated frame of reference. We will refer to this process as realignment and conceive of it as involving the alignment of the secondary reference frame associated with the allocentric viewpoint with the primary reference frame associated with the participant's observer-centered viewpoint (Presson \& Montello, 1994; Roskos-Ewoldsen, McNamara, Shelton, \& Carr, 1998; Sholl, 1995). This viewpoint alignment hypothesis implies that the misalignment effect should decrease when realignment is facilitated-for example, by providing an advance cue that specifies the viewpoint for a subsequent judgment. The aim of the present study was to examine the effect of an advance viewpoint cue when people make a spatial judgment (e.g., to identify the relative location, such as left or right, of a target from a reference viewpoint that could be different from the observer's viewpoint). 
One question in the present study is whether participants can effectively use advance viewpoint information to overcome the costs associated with realignment. Previous research has suggested that it may be difficult or impossible to effectively use advance viewpoint cues to establish allocentric viewpoints. For example, Huttenlocher and Presson (1973) suggested that an individual performing a perspective task "can imagine the array in some other position than its present one, but he cannot imagine himself in some other position than his present one" (p. 298). Using several tasks similar to the spatial judgment task in the present study, Hintzman, O'Dell, and Arndt (1981) also found that advance information about viewpoints did not reduce misalignment effects or provide any overall performance benefit relative to advance information about a spatial target.

Roskos-Ewoldsen et al. (1998, Experiment 2) attempted to measure realignment time independently of response time by providing advance information about imagined viewpoints to be adopted for a pointing task in a remembered spatial layout, allowing participants to indicate when they had adopted a viewpoint and were ready for a target. They found that these realignment times showed a misalignment effect that increased with the discrepancy of allocentric and observer-centered viewpoints, suggesting that the realignment process can be based on an advance cue. However, latencies for the subsequent pointing responses also showed a misalignment effect. This result suggests that the participants' efforts to adopt an imagined viewpoint in advance were ineffective, perhaps because they could not maintain these imagined viewpoints. However, Roskos-Ewoldsen et al.'s study did not allow an assessment of the effectiveness of advance viewpoint information relative to an uncued condition. Furthermore, the participants in their study made judgments about a remembered space, and the very long pointing latencies that were observed (on average, greater than $10 \mathrm{sec}$ ) suggest that the combination of realignment and judgment processes in their task placed stress on the participants' working memory capacity.

In the present experiments, we compared spatial judgment performance when an advance cue specified either target location or allocentric viewpoint. In this task, participants make judgments about the target location from a particular viewpoint, combining left-right and near-far dimensions that are relative to that viewpoint. Both target and viewpoint are necessary to make a spatial judgment, but it is the relation between the allocentric and the observer-centered viewpoints that constitutes misalignment of reference frames. Like Roskos-Ewoldsen et al. (1998), we expected that viewpoint information alone could engage realignment processes. Furthermore, although advance cues specifying either viewpoint or target reduce the amount of information that must be processed when the full display appears, we expected that advance viewpoint information, but not advance target location information, would reduce the misalignment effect.
A second question in the present study was whether the misalignment effect has another source, in addition to the costs of realignment. The disparity between allocentric and observer-centered viewpoints often results in conflicting responses. Imagine someone sitting across from a friend at the dinner table. That person would ask the friend to "pass the dish on the right," although the dish is in fact on his or her "left." As such, labeling a spatial relation from an allocentric viewpoint may create a conflict with response codes from the observer-centered viewpoint (Huttenlocher \& Presson, 1979), and extra time may be required to resolve the conflict. In Experiment 1, we tested whether the misalignment effect could be reduced by advance viewpoint information. In Experiment2, we tested whether the misalignment effect could be attributed to response conflict.

The paradigm adopted here included several features that we thought would contribute to effective use of advance information about realignment. First, a representation of the spatial workspace was always visible throughout a trial, and this representation included the locations of both the reference viewpoint and the spatial target. This contrasts with standard perspective tasks, in which the reference viewpoint is outside the spatial array (Huttenlocher $\&$ Presson, 1973; Presson, 1982). Second, we described this workspace to the participants as a human-scaled spacea table-in which the presence of multiple possible perspectives would be familiar. Third, the elements composing the spatial array varied from trial to trial. Finally, advance viewpoint cues specified an imagined individual whose identity remained constant throughout the experiment but whose location and, thus, viewpoint changed from trial to trial. Taken together, these aspects of the task should encourage and support a strategy in which participants adopt an allocentric viewpoint to perform the task, rather than other strategies, such as mentally rotating the display into alignment with their observer-centered frame of reference (Hinton \& Parsons, 1981; Presson, 1982).

A sample display used in the present study is shown in Figure 1A. The ellipse was introduced as a table around which five people were seated and facing the center. One of the five names-say, Annie-served as a reference person and appeared on every trial in a randomly chosen location that specified the allocentric viewpoint for that trial. On each trial, the remaining four names were randomly selected from a pool of 20 different names. The participants' task was to make a spatial judgment (e.g., near-left, nearright, far-left, or far-right) regarding the relative location of a target person from the viewpoint of the reference person. The target location varied trial by trial. We asked the participants to make these judgments as quickly as possible while maintaining a high level of accuracy.

As in Figure 1B, the viewpoint at the bottom center of the display preserves much of the reference frame associated with the participant's viewpoint, as do photographs and perspectival drawings of scenes, in that the bottom center depicts the location nearest the camera's or the artist's 


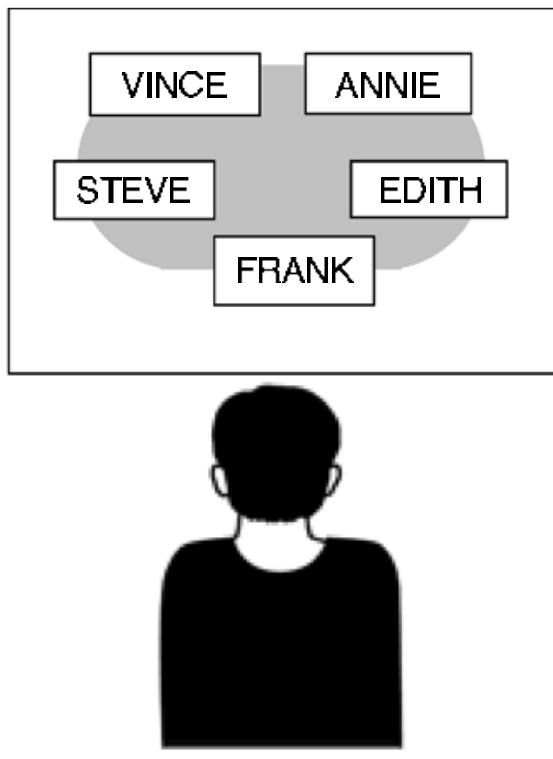

(A)

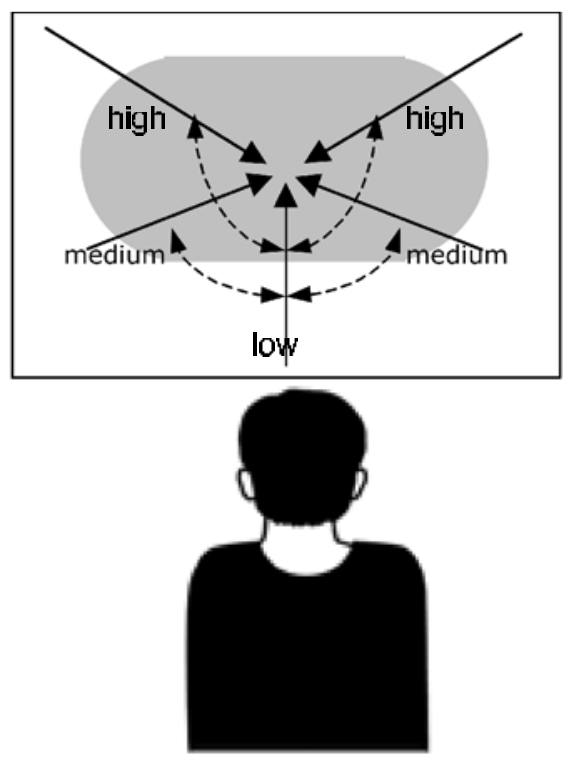

(B)

Figure 1. Sample display for a viewpoint-relative spatial judgment task (A) and angular disparities of allocentric viewpoints (B).

viewpoint. Therefore, the extent of misalignment between allocentric and observer-centered viewpoints is defined by the angle formed by the bottom center, the center of the table, and the currently relevant allocentric viewpoint. Misalignment is, therefore, low when the reference person is at the bottom position, medium when at the lowerleft or lower-right position, and high when at the upperleft or upper-right position. The misalignment effect is, therefore, defined as the performance deficit (increased response latency or lower accuracy) associated with allocentric viewpoints at the lower or upper positions, as opposed to the viewpoint at the bottom position.

\section{EXPERIMENT 1}

Experiment 1 tested whether advance processing of a viewpoint location would decrease the misalignment effect. To allow differential advance processing of either viewpoint or target locations, we used five stimulus onset asynchronies (SOAs) - 0, 200, 400, 600, and $800 \mathrm{msec}-$ between the advance information and the rest. On advance viewpoint trials, the realignment process should begin with the display of the viewpoint location, reducing the misalignment effect with increases in SOA. On advance target trials, however, realignment could not be initiated during the SOA, and the misalignment effect should not depend on SOA.

\section{Method}

Stimulus. The displays were prepared and presented using the Micro Experimental Lab system. The ellipse was constructed by placing two identical circles side by side and superimposing a square whose side was equal to the diameter of the circles. The names were picked from 20 different five-letter names.

Procedure and Design. The participants were told that the reference person would be the same throughout the experiment, whereas the target person would change trial by trial. As in Figure 2, each trial began with a ready message at the center of the screen. When a participant pressed the space bar, an ellipse with the target name for the trial replaced the ready message. The participants studied the name as long as they wanted, then pressed the space bar. The target name then disappeared from the center of the display, and either the target or the reference name appeared at its location for the trial. The target location was displayed in advance on half of the trials, and the viewpoint location was displayed on the other half. Following an SOA of $0,200,400,600$, or $800 \mathrm{msec}$, the remaining names appeared in their locations. On the 0 -sec SOA trials, of course, all the names appeared simultaneously, but these trials were assigned to either advance target or advance viewpoint conditions on the basis of the randomization scheme. Advance information about either target or viewpoint location in principle allowed the participants to calculate in advance the appropriate response for each of the four remaining locations, in which the other critical piece of information might appear. Response uncertainty given the advance cue was thus equal for the advance target and the advance viewpoint conditions.

The location of the allocentric viewpoint corresponded to one of three levels of misalignment: low, medium, and high. The target locations relative to the viewpoint were defined as near-left, far-left, near-right, and far-right, for which "X," "Z," ".," and "/" keys were assigned, respectively. In Figure 1, for example, the target "Vince" is to the near-right of the reference person "Annie." Left index and middle fingers were assigned to the " $X$ " and " $Z$ " keys, and right index and middle fingers were assigned to the "." and "/" keys, respectively, providing a spatially compatible mapping relative to the observer-centered viewpoint.

The type of advance information was manipulated across blocks. There were 100 trials in each of eight blocks, 20 consecutive trials with each SOA. The order of SOAs in a block was randomized, with the constraint that the 0 -msec SOA trials were not presented at the 


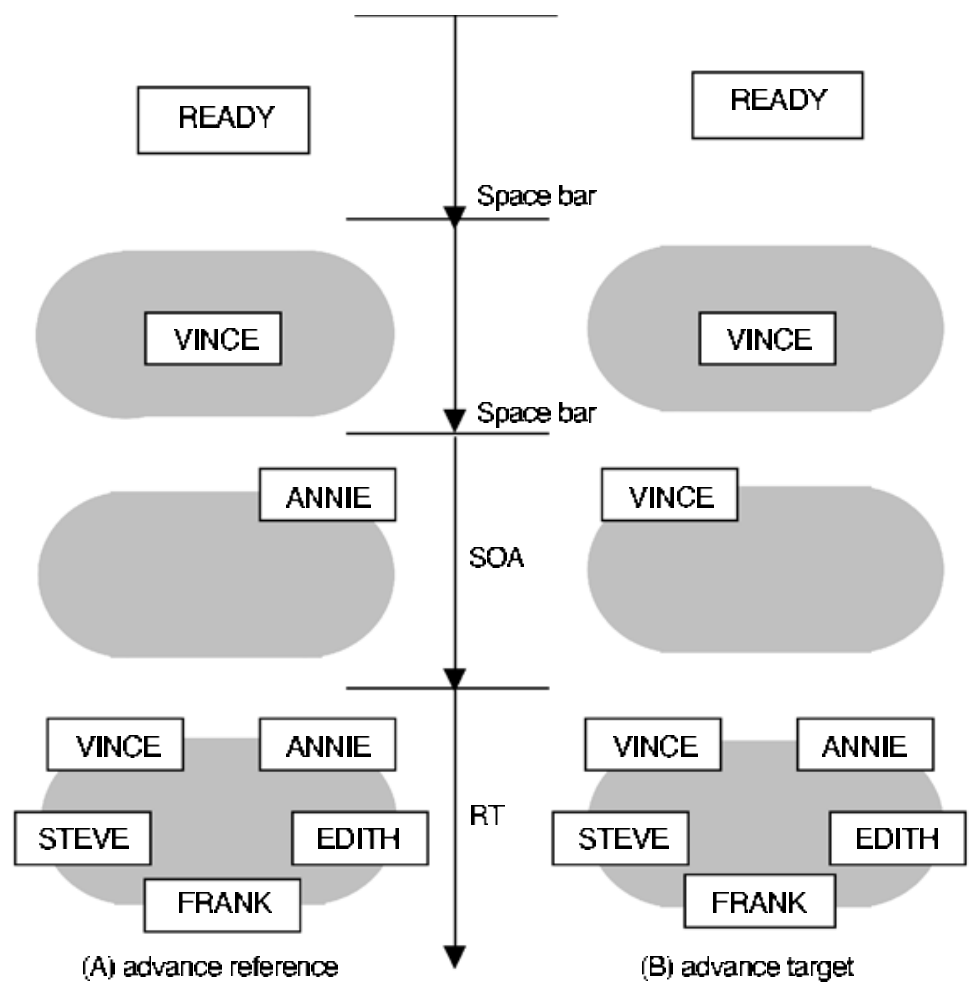

Figure 2. Sequence of events for a trial with advance viewpoint information (A) and a trial with advance target information (B). In the no advance information trials, all the names appeared simultaneously.

beginning of a block so that the serial nature of the presentation would be salient. Advance viewpoint and advance target blocks alternated, and their order was counterbalanced over participants. The design was a 2 (advance information) $\times 3$ (alignment) $\times 5(\mathrm{SOA})$ within-subjects factorial.

Participants. Twenty-eight college students from introductory psychology classes at Pennsylvania State University participated in return for extra course credit.

\section{Results and Discussion}

For both accuracy and latency, three-way analyses of variance (ANOVAs) were conducted, with the type of advance information (viewpoint and target), misalignment (low, medium, and high), and SOA (0, 200, 400, 600, and $800 \mathrm{msec}$ ) as variables. One participant, whose accuracy
(.23) was near the chance level of .25 correct, was excluded from the analyses below. Statements about differences among means in both experiments are based on Neuman-Keuls comparisons with $p=.05$.

Accuracy. Table 1 presents mean accuracies from all conditions. Accuracy was higher with advance target information (.87) than with advance viewpoint information $\left[.85 ; F(1,26)=5.65, M S_{\mathrm{e}}=0.017, p<.05\right]$. The main effect of misalignment was significant $[F(2,52)=26.75$, $\left.M S_{\mathrm{e}}=0.016, p<.0001\right]$. Accuracy was higher with the low misalignment (.91) than with medium (.84) and high (.84) misalignments. Accuracy did not differ between the medium and the high misalignments. Accuracy also varied with SOA $\left[F(4,104)=8.40, M S_{\mathrm{e}}=0.008, p<.0001\right]$.

Table 1

Mean Accuracy for Each Condition in Experiment 1 (With Standard Errors)

\begin{tabular}{|c|c|c|c|c|c|c|c|c|c|c|c|c|}
\hline \multirow{4}{*}{$\begin{array}{c}\text { Stimulus Onset } \\
\text { Asynchrony }\end{array}$} & \multicolumn{12}{|c|}{ Misalignment } \\
\hline & \multicolumn{6}{|c|}{ Advance Viewpoint } & \multicolumn{6}{|c|}{ Advance Target } \\
\hline & \multicolumn{2}{|c|}{ Low } & \multicolumn{2}{|c|}{ Medium } & \multicolumn{2}{|c|}{ High } & \multicolumn{2}{|c|}{ Low } & \multicolumn{2}{|c|}{ Medium } & \multicolumn{2}{|c|}{ High } \\
\hline & $M$ & $S E$ & $M$ & $S E$ & $M$ & $S E$ & $M$ & $S E$ & $M$ & $S E$ & $M$ & $S E$ \\
\hline 0 & .87 & .03 & .80 & .03 & .80 & .03 & .89 & .03 & .81 & .03 & .81 & .03 \\
\hline 200 & .90 & .03 & .84 & .03 & .82 & .03 & .94 & .02 & .83 & .03 & .85 & .03 \\
\hline 400 & .90 & .03 & .86 & .03 & .85 & .03 & .94 & .02 & .87 & .02 & .87 & .03 \\
\hline 600 & .90 & .03 & .86 & .02 & .85 & .03 & .94 & .01 & .86 & .02 & .87 & .02 \\
\hline 800 & .89 & .03 & .85 & .03 & .82 & .03 & .95 & .01 & .85 & .03 & .84 & .03 \\
\hline
\end{tabular}




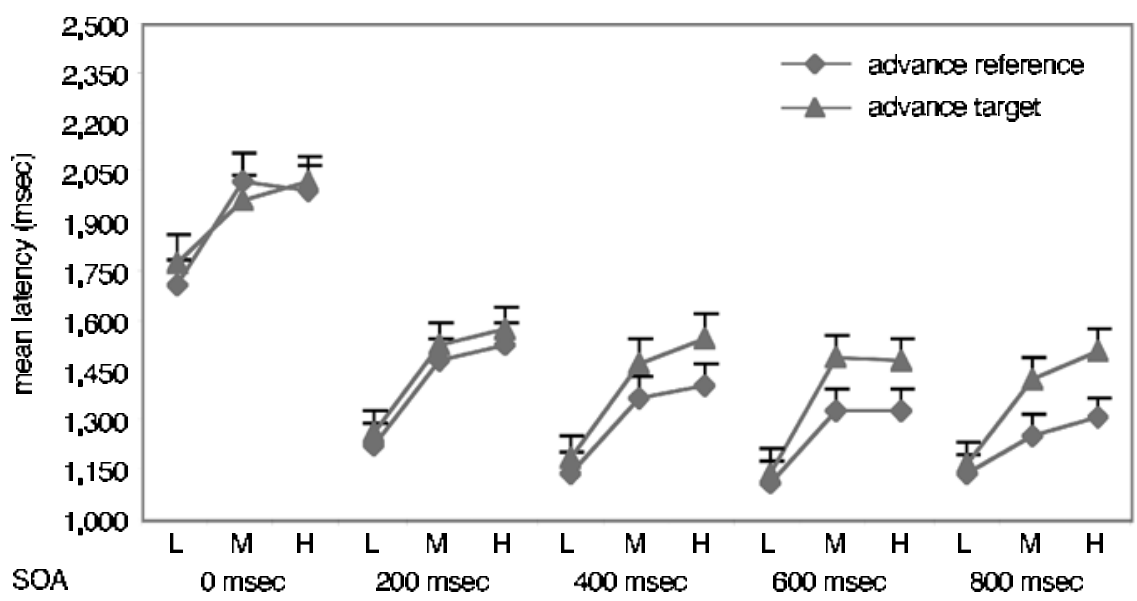

Figure 3. Mean judgment latency as a function of type of advance information for each misalignment and stimulus onset asynchrony (SOA) in Experiment 1 . The letters $\mathrm{L}$, M, and $\mathrm{H}$ indicate low, medium, and high angular disparities, respectively.

Accuracy was lower with the 0 -msec SOA (.84) than with the 200-msec SOA (.86), which did not differ from accuracies with the 400-(.88), 600-(.88), and 800-msec (.87) SOAs. No interactions were significant $(p>.10)$.

Latency. Figure 3 plots latency as a function of SOA, alignment, and advance information. The participants responded more quickly with advance viewpoint information $(1,427 \mathrm{msec})$ than with advance target information $\left[1,506 \mathrm{msec} ; F(1,26)=14.34, M S_{\mathrm{e}}=88,480, p<.01\right]$, consistent with the hypothesis that the realignment process could begin with viewpoint information only. Although the higher accuracy with advance target information raises the possibility of a speed-accuracy tradeoff, the overall pattern of results suggests no further associations of longer latencies with lower accuracy. The main effect of misalignment was significant $\left[F(2,52)=44.69, M S_{\mathrm{e}}=\right.$ $145,064, p<.0001]$. Latencies were shorter with low misalignment $(1,289 \mathrm{msec})$ than with medium $(1,538 \mathrm{msec})$ and high $(1,573 \mathrm{msec})$ misalignments. Latencies did not differ between the medium and the high misalignments. Latencies decreased with longer SOAs $[F(4,104)=238.35$, $\left.M S_{\mathrm{e}}=45,244, p<.0001\right]$. Latencies were longest with the 0 -msec SOA $(1,918 \mathrm{msec})$, intermediate with the $200-\mathrm{msec}$ SOA $(1,436 \mathrm{msec})$, and shortest with the 400 $(1,358 \mathrm{msec}), 600-(1,318 \mathrm{msec})$, and $800-\mathrm{msec}(1,303 \mathrm{msec})$ SOAs. The type of advance information interacted with misalignment $\left[F(2,52)=5.43, M S_{\mathrm{e}}=15,941, p<.01\right]$ and with SOA $\left[F(4,104)=7.61, M S_{\mathrm{e}}=13,945, p<.0001\right]$.

Most important, the three-way interaction involving advance information, misalignment, and SOA was significant $\left[F(8,208)=3.40, M S_{\mathrm{e}}=14,112, p<.01\right]$. This threeway interaction is what the viewpoint alignment hypothesis would predict. Advance processing of the viewpoint should have facilitated realignment, therefore reducing the misalignment effect with increases in SOA. However, advance processing of the target would not result in such fa- cilitation. To verify this pattern, we conducted a two-way ANOVA with misalignment and SOA as variables for each type of advance information. The interaction between misalignment and SOA was significant with advance reference information $\left[F(8,208)=2.98, M S_{\mathrm{e}}=14,279, p<\right.$ .01 ], but not with advance target information $(p>.05)$. With advance viewpoint information, the latency deficit of medium misalignment, as compared with low misalignment, was greater with the $0-\mathrm{msec}$ SOA $(318 \mathrm{msec})$ than with the $800-\mathrm{msec}$ SOA $(178 \mathrm{msec})$. The latency deficit of high misalignment was greater with the 200 -msec SOA (302 msec) than with the $800-\mathrm{msec}$ SOA $(171 \mathrm{msec})$.

This facilitation with advance viewpoint information supports the idea that realignment is critical in this task. If the misalignment effect is due solely to the realignment process, however, this effect should disappear with advance viewpoint with a sufficiently long SOA. However, although reduced, the simple main effect of misalignment was significant in all combinations of SOAs and the type of advance information, including the 800-msec SOA $(p \mathrm{~s}<$ $.01)$. One explanation for this is that even $800 \mathrm{msec}$ was not enough to complete the realignment process and fully establish an allocentric viewpoint. Although the means plotted in Figure 3 suggest that performance on advance viewpoint trials was approaching asymptote, we cannot rule out this possibility.

\section{EXPERIMENT 2}

Experiment 2 tested the possibility that the misalignment effect is due partly to response conflict. According to some spatial stimulus-response compatibility theories, presentation of a stimulus at a spatial location automatically generates a spatial code in relation to the body position (Kornblum, Hasbroucq, \& Osman, 1990). For example, if a target appears on the participant's left, the left 
response is activated. However, this automatically activated response code may or may not correspond with the correct answer in a particular task. In a situation such as the one in Experiment 1, the allocentric viewpoint located at the bottom position does not create response conflict, because its left-right axis corresponds with that of the participants' observer-centered viewpoint. However, allocentric viewpoints at other locations could produce response conflicts. For example, some left responses were made to the locations that were actually on the right-hand side of the participants, and vice versa. Therefore, the correct response with the instructed allocentric viewpoint may conflict with the natural response to the target. The response conflict hypothesis suggests that this conflict is responsible, in part, for the misalignment effect.

We tested the response conflict hypothesis by using two types of mappings between the response code automatically activated on the basis of the participant's viewpoint and the response code generated on the basis of instructions. In the spatial response condition, the instructed response codes were spatial (near-left, etc.), as in Experiment 1 , so that they were spatially compatible with the participant's observer-centered viewpoint. In the arbitrary response condition, the instructed response codes were not spatial but were letters (W, S, L, and P), and corresponding keys on the computer keyboard were used as response keys. Each letter was arbitrarily assigned to an allocentric-relative target location. To describe the appropriate letter responses, we showed the participants a pictorial depiction of the response arrangement with each misalignment view and explained the situation while avoiding spatial terms such as near-left or far-right. Thus, although it is still possible that the participants in the arbitrary response condition may have generated spatial response codes and translated them into the corresponding letter response codes, the spatial arrangement of response keys could not provide spatial compatibility between the instructed response code and an automatically activated response code.

We reasoned that response conflict should be greater with spatial responses than with arbitrary responses, because target presentation will automatically activate spatial response codes (Kornblum et al., 1990), but not alphabetic response codes. Arbitrary mapping may reduce response conflict in two ways. First, because it is arbitrary, it will slow down response generation. The additional time required for response generation may allow automatically activated spatial responses to decay or to be inhibited. Second, arbitrary mapping produces explicit responses (e.g., $\mathrm{P}$ or $\mathrm{W}$ ) that should not conflict with automatically activated responses that are spatial in nature. Therefore, if, in part, the misalignment effect reflects response conflict, it should be greater in the spatial response condition.

In Experiment 2, a single 500-msec SOA was used for advance viewpoint and advance target information, because in Experiment 1 latency seemed to drop rapidly from a 0 -msec SOA to a 200 -msec SOA but declined little beyond a 400-msec SOA with either type of advance information. Along with the advance viewpoint and target conditions, we also included the no advance information condition, in which the SOA was 0 msec. As in Experiment 1 , either type of advance information might speed processing by providing a warning signal or by reducing search demands (Hintzman et al., 1981), because with no advance information, a participant must search for two names among five, whereas the search could be limited to finding one among four names with either type of advance information. With regard to response conflict, because the final response code can be generated only after both viewpoint and target locations become available, the extent of competition does not depend on the type of advance information. Therefore, the type of response mapping should not interact with the type of advance information. However, the type of response mapping should interact with the misalignment effect, so that the misalignment effect will be greater with spatial than with arbitrary response mappings.

\section{Method}

Procedure and Design. The procedure was similar to that in Experiment 1, with several changes. The type of advance information was randomized rather than blocked, as in Experiment 1. This provided a check on the possibility that the results of Experiment 1 might have been due, in part, to the adoption of different strategies in the advance viewpoint and the advance target conditions. Because the results showed no evidence of this possibility, it will not be discussed further.

The participants in the arbitrary condition could request help on the response mappings during the practice block by pressing the "Q" key anytime during the trial to display the pictorial representation of the mapping. The help display appeared on the upper right corner of the screen and disappeared at the end of the trial. The assignment of letter to relative location was random across participants, with the constraint that the overall response mapping was not spatially congruent for any participant. There were six (one practice and five main) blocks, and each block contained 60 trials resulting from the combinations of three types of advance information (none, viewpoint, and target), five viewpoint locations, and four relative locations of the target. These 60 trials appeared in a random sequence, with a different sequence for each block. The resulting experimental design was a 2 (response code) $\times 3$ (advance information) $\times 3$ (alignment) mixed factorial.

Participants. Sixty-one college students (20 in the spatial response and 41 in the arbitrary response conditions) from introductory psychology classes at Pennsylvania State University participated in return for extra course credit. Across participants in the arbitrary response condition, all possible mappings of response keys to relative locations were used, except the fully congruent mapping ( $\mathrm{W}=$ far-left, $S=$ near-left, $\mathrm{L}=$ near-right, and $\mathrm{P}=$ far-right), for a total of 23 mappings. Most of these mappings were replicated over 2 participants, but owing to participant availability, only 41 individuals participated, so that several mappings were performed by only 1 participant.

\section{Results and Discussion}

For both accuracy and latency, we conducted three-way ANOVAs with response code (spatial or arbitrary), advance information (viewpoint, target, or none), and misalignment (low, medium, or high) as factors. Four participants from the arbitrary condition, whose accuracies were at or below the chance level of .25, were excluded from the analyses. 
Table 2

Mean Accuracy for Each Condition in Experiment 2 (With Standard Errors)

\begin{tabular}{|c|c|c|c|c|c|c|c|c|c|c|c|c|}
\hline \multirow[b]{3}{*}{ Advance Information } & \multicolumn{6}{|c|}{ Spatial Response } & \multicolumn{6}{|c|}{ Arbitrary Response } \\
\hline & \multicolumn{2}{|c|}{ Low } & \multicolumn{2}{|c|}{ Medium } & \multicolumn{2}{|c|}{ High } & \multicolumn{2}{|c|}{ Low } & \multicolumn{2}{|c|}{ Medium } & \multicolumn{2}{|c|}{ High } \\
\hline & $M$ & $S E$ & $M$ & $S E$ & $M$ & $S E$ & $M$ & $S E$ & $M$ & $S E$ & $M$ & $S E$ \\
\hline Viewpoint & .96 & .01 & .86 & .02 & .84 & .02 & .88 & .02 & .84 & .02 & .85 & .02 \\
\hline Target & .98 & .01 & .88 & .01 & .86 & .03 & .89 & .02 & .85 & .02 & .84 & .03 \\
\hline None & .93 & .01 & .82 & .03 & .82 & .02 & .85 & .02 & .82 & .02 & .79 & .02 \\
\hline
\end{tabular}

Accuracy. Table 2 presents mean accuracies from all conditions. Accuracy was higher with either advance viewpoint (.87) or target (.88) information than with no advance information $(.83)\left[F(2,110)=19.71, M S_{\mathrm{e}}=0.005\right.$, $p<.0001]$. Accuracy was higher with low misalignment (.90) than with medium (.84) and high (.83) misalignments $\left[F(2,110)=47.55, M S_{\mathrm{e}}=0.006, p<.0001\right]$. Accuracy did not differ between medium and high misalignments. The interaction between response code and misalignment was significant $\left[F(2,110)=11.57, M S_{\mathrm{e}}=\right.$ $0.006, p<.0001]$. As was predicted, this interaction was due to greater misalignment effects (accuracy difference of medium or high misalignments and low misalignment) in the spatial response code condition than in the arbitrary response condition. The accuracy deficit for medium misalignment was greater in the spatial response condition $(M=.10, S E=.016)$ than in the arbitrary condition $[M=$ $.04, S E=.009 ; t(55)=3.66, p<.01]$. The accuracy deficit for high misalignment was greater in the spatial response condition $(M=.12, S E=.02)$ than in the arbitrary condition $[M=.04, S E=.01 ; t(55)=3.7, p<.01]$. No other effects were significant $(p>.20)$.

Latency. Figure 4 plots latency as a function of alignment and the type of advance information in each response mapping condition. Latencies were shortest with advance viewpoint information $(1,750 \mathrm{msec})$, intermediate with advance target information $(1,854 \mathrm{msec})$, and longest with no advance information $[2,356 \mathrm{msec} ; F(2,110)=540.85$, $\left.M S_{\mathrm{e}}=29,866, p<.0001\right]$. Latencies were shorter with low $(1,840 \mathrm{msec})$ than with medium $(2,054 \mathrm{msec})$ or high $(2,066 \mathrm{msec})$ misalignments $\left[F(2,110)=49.11, M S_{\mathrm{e}}=\right.$ $63,247, p<.0001]$. Latencies did not differ between the medium and the high misalignments. The interaction between advance information and misalignment was significant $\left[F(4,220)=2.97, M S_{\mathrm{e}}=19,543, p<.05\right]$. More important, supporting the response conflict hypothesis, the interaction between type of response code and misalignment was significant $\left[F(2,110)=5.61, M S_{\mathrm{e}}=63,247, p<\right.$ .01]. The misalignment effect (latency difference) for the medium misalignment was greater in the spatial response condition $(M=315 \mathrm{msec}, S E=65 \mathrm{msec})$ than in the arbitrary condition $[M=165 \mathrm{msec}, S E=34 \mathrm{msec}$; $t(55)=$ $2.25, p<.05]$. The misalignment effect for the high misalignment was greater in the spatial response condition $(M=342 \mathrm{msec}, S E=67 \mathrm{msec})$ than in the arbitrary condition $[M=163 \mathrm{msec}, S E=32 \mathrm{msec} ; t(55)=2.73, p<.01]$.

Both accuracy and latency showed that the misalignment effect was greater with spatial responses than with arbitrary responses, whereas the misalignment effect was substantial in both conditions. As was predicted, arbitrary responses lengthened latencies, possibly minimizing the effect of automatic activation of spatial codes for the tar-

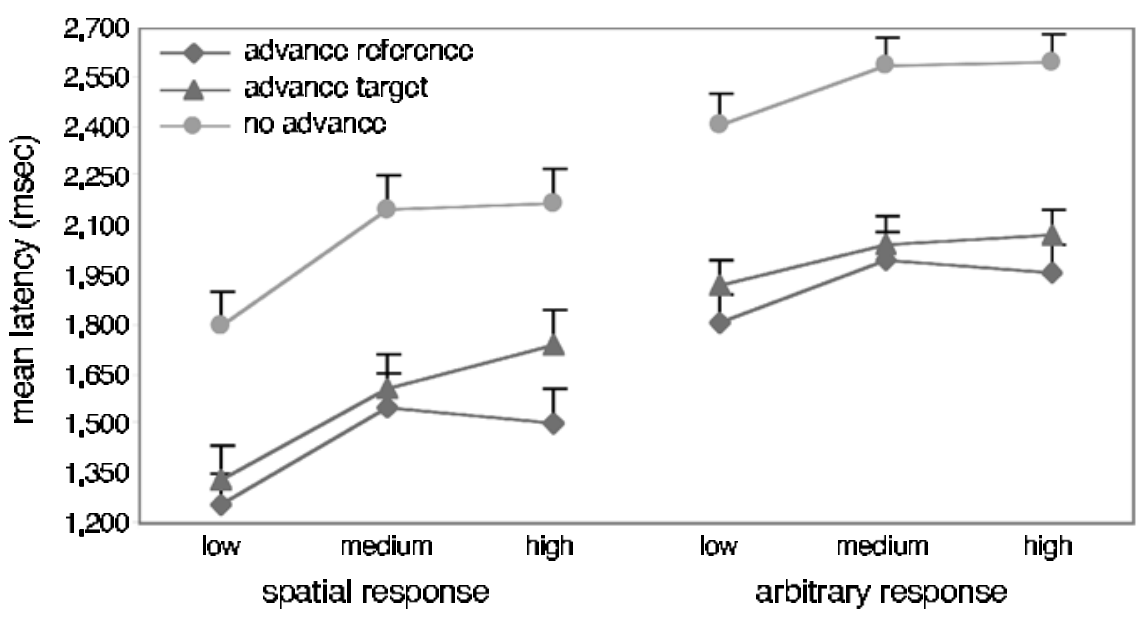

Figure 4. Mean judgment latency as a function of type of advance information for each misalignment in Experiment 2. 
get location. Also, in Experiment 2, advance viewpoint information resulted in a greater benefit than did advance target information, indicating that viewpoint alignment is indeed critical for performing spatial judgments with allocentric viewpoints. These results support the hypothesis that the misalignment effect may reflect both viewpoint alignment and response conflict. The advance target information also resulted in substantially shorter latencies, as compared with the no advance information condition, although they were longer than those in the advance viewpoint condition. At least part of this general advance information benefit can be attributed to a reduction in the visual search demands of the task.

\section{GENERAL DISCUSSION}

The viewpoint alignment hypothesis implies that the misalignment effect reflects the realignment process required to establish an allocentric viewpoint. The response conflict hypothesis implies that the misalignment effect is due in part to competition between response codes based on allocentric and observer-centered viewpoints. In Experiment 1 , the misalignment effect was reduced with advance viewpoint information, but not with advance target location information, supporting the viewpoint alignment hypothesis. In Experiment 2, the misalignment effect was greater with spatial responses than with arbitrary responses regardless of advance information, whereas the misalignment effect itself was significant in both mapping conditions. This is consistent with the response conflict hypothesis. The present study thus demonstrates that allocentric viewpoints can be successfully cued and suggests that the misalignment effect may have two sources, viewpoint alignment and response conflict. The two hypotheses are not mutually exclusive. Viewpoint alignment may take place early, as reflected in the reduced misalignment effect with advance viewpoint information. In contrast, response conflict may take place later, because response conflict can arise only after both viewpoint and target locations are available and response codes based on both observercentered and allocentric viewpoints have been activated. The possibility of this dual-locus account of the misalignment effect is one contribution of the present study.

\section{Viewpoint Alignment}

Issues of viewpoint alignment are involved in many spatial judgment tasks, ranging from handedness judgments (e.g., Hinton \& Parsons, 1981; Shepard \& Metzler, 1972) and classic perspective tasks (e.g., Presson, 1982) to studies of spatial knowledge of maps and environments (e.g., Roskos-Ewoldsen et al., 1998; Sholl, 1995). In a number of such tasks, investigators have found that advance cuing of allocentric viewpoints or non-observercentered frames of reference is ineffective, and some authors have suggested that it is not possible to use the advance cue (e.g., Hintzman et al., 1981; Huttenlocher \& Presson, 1973; Shepard \& Hurwitz, 1984). The present study, in contrast, provides evidence that advance information about an allocentric viewpoint can be effectively used, speeding spatial judgments and reducing the misalignment effect. In demonstrating these effects, the present study extends previous demonstrations that individuals do use advance cues to initiate realignment processes (Roskos-Ewoldsen et al., 1998). This result is important, in part, because it provides direct support for the viewpoint alignment account of misalignment effects in spatial judgment. A consideration of the nature of the present task also suggests some possible conditions for effective cuing of allocentric perspectives. As has been described above, the present paradigm involved cuing a perspective associated with an individual whose location was included in a human-scaled spatial array, with a continuously available display representing that spatial array. Furthermore, the task likely discouraged the object rotation strategy in which individuals might rotate a spatial array. The present experiments did not directly compare object rotation with viewpoint rotation (cf. Wraga, Creem, \& Proffitt, 1999). However, we suggest that the effectiveness of cuing allocentric viewpoints lends support to the view that mentally establishing an allocentric viewpoint is a strategy available in many spatial judgment situations, although some paradigms may make this strategy difficult to use (e.g., Eley, 1988; Hinton \& Parsons, 1981; Presson, 1982).

\section{Response Conflict}

Response conflict is a well-established explanatory mechanism in a variety of reaction time tasks (see Eriksen, 1995 , for a review). The basic idea is that processing is slowed when a display automatically activates a response code that conflicts with the response code that is correct according to the task instructions, because extra time is required to resolve the conflict and produce the correct response. In spatial stimulus-response compatibility tasks, participants respond to the location of a stimulus, using instructed stimulus-response mappings that are compatible or incompatible. For example, if the stimulus appears to the left side of a display, the left response is compatible with the stimulus, but not the right response. When participants are instructed to use incompatible response mappings, response times are longer. Such incompatible mappings are analogous to a spatial judgment situation in which an individual is asked to make a left-right judgment from the viewpoint of someone sitting across a table. For example, in Figure 1, Vince is to the observer's left but to Annie's right. We might, therefore, expect response conflict to play a role in explaining the misalignment effect in spatial judgment, just as it plays an important role in theories of spatial stimulus-response compatibility effects (De Jong, Liang, \& Lauber, 1994). That is, the automatically activated response facilitates a compatible response but interferes with an incompatible response. In our experiments, the spatial response codes could potentially result in response conflicts, because the response keys were also spatially arranged. However, the arbitrary or nonspatial response 
codes would not have resulted in a response conflict. The misalignment effect was greater when appropriate response codes were spatial than when they were not, strongly suggesting that the misalignment effect may be partly due to response conflict.

\section{Alternative Accounts and Limitations}

Alternative explanations in terms of a single mechanism, instead of a dual-locus mechanism, cannot explain the misalignment effect in the present study. First, an account solely in terms of response conflict would predict no difference between advance viewpoint and advance target location information. Moreover, this cannot explain either the overall benefit or the reduction in the misalignmenteffect with advance viewpoint information. Second, an account solely in terms of viewpoint alignment would predict that the misalignment effect should disappear with advance viewpoint information. This can explain the reduced misalignment effect with arbitrary response mappings only with the assumption that realignment is a very slow process that can be completed in the longer time frame than the one associated with arbitrary mappings. Therefore, we argue that these are not as convincing as the dualmechanism explanation (both viewpoint alignment and response conflict) that we propose here.

An alternative to the response conflict hypothesis might point to the continued availability of the observer-centered viewpoint as the source for the remaining misalignment effect, a possibility also suggested by Roskos-Ewoldsen et al. (1998) in considering the failure of their procedure to completely separate realignment and judgment processes. After all, the observer-centered viewpoint must be used to read the (canonically oriented) names in search of the viewpoint and target locations. Therefore, the observercentered frame of reference might have been reinstated as soon as the entire display had been filled with names, requiring processing of the allocentric viewpoint. This possibility might exacerbate the viewpoint conflict on advance target trials, because the allocentric reference frame cannot be established until the full display is available, thus resulting in a greater misalignment effect. This alternative explanation assumes that conflict is more detrimental when a reference frame is established than when an already established one is maintained. Although the data do not rule out this possibility, it fails to explain the reduced misalignment effect when a spatially arbitrary response mapping is used, as in Experiment 2. Because the reinstatement process should not depend on the type of response code or mapping, it is hard for this account to explain the interaction between response code and misalignment.

There are several concerns in interpreting the present results. First, Experiment 1 sampled a relatively narrow range of SOAs. Although response times declined little as SOA increased beyond $400 \mathrm{msec}$, it is possible that the misalignment effect would decline further with longer SOAs. However, if there is indeed a response conflict component in the misalignment effect, even very long
SOAs might not eliminate the effect. This is why we attempted to test the response conflict hypothesis directly in Experiment 2, instead of sampling longer SOAs.

Second, although the longer response latency was predicted in the arbitrary mapping condition, one might argue that the large latency difference between the spatial response and the arbitrary response conditions suggests caution in interpreting the interaction. However, because this interaction was due to a reduced misalignment effect with a slower response latency, any accounts based only on viewpoint realignment would require highly specific assumptions about the time course of the realignment. Moreover, it is not clear why the realignment process should depend on whether the response codes are spatial or not. Therefore, we prefer the dual-locus account, on the basis of both viewpoint realignment and response conflict. We acknowledge the importance of further investigating the circumstances in which viewpoint alignment and response conflict affect the difficulty of making spatial judgments.

Another concern is that our experimental procedures made it easier to process the viewpoint name than the target names because the viewpoint name was constant but the target name varied trial by trial. Also, the target name was presented initially at the center of the ellipse and then was presented at one of the locations around the ellipse. However, time to study the target name was under the participants' control, and the search demands were equivalent in the advance viewpoint and the advance target cases. More important, although faster processing of the viewpoint name might explain a main effect of type of advance information, we do not see how it could explain the observed reduction in the misalignment effect with advance viewpoint information.

Finally, the misalignment effect in the present study may differ in nature from that found in other studies. In both experiments, the medium and the high misalignment viewpoints did not differ from each other. The misalignment effect has often been found to be a function of the extent of misalignment (e.g., Wraga et al., 1999). Although a linear misalignment effect would be a more straightforward result, we believe that the lack of difference between the medium and the high misalignments does not compromise our claim for a dual mechanism for the misalignment effect.

\section{Conclusion}

The present results suggest that misalignment effects in spatial judgment may result from both viewpoint alignment and response conflict. Although the present data do not definitively rule out all alternative accounts, we believe that they point to the need to consider both of the possible mechanisms in understanding spatial judgment. How and when a particular source will contribute may depend on whether the task supports a viewpoint alignment strategy and on whether conflicting spatial responses are automatically activated by the displays. Examining these possibilities provides a number of directions for future research. 


\section{REFERENCES}

CoOper, L. A., \& SHEPARD, R. N. (1973). The time required to prepare for a rotated stimulus. Memory \& Cognition, 1, 246-250.

De Jong, R., LiAnG, C.-C., \& LAUber, E. (1994). Conditional and unconditional automaticity: A dual-process model of effects of spatial stimulus-response correspondence. Journal of Experimental Psychology: Human Perception \& Performance, 20, 731-750.

ELEY, M. G. (1988). Determining the shapes of land surfaces from topographical maps. Ergonomics, 31, 355-376.

ERIKSEN, C. W. (1995). The flankers task and response competition: A useful tool for investigating a variety of cognitive problems. Visual Cognition, 2, 101-118.

Hinton, G. E., \& PARSONs, L. M. (1981). Frames of reference and mental imagery. In J. [B.] Long \& A. [D.] Baddeley (Eds.), Attention and performance IX (pp. 261-277). Hillsdale, NJ: Erlbaum.

Hintzman, D. L., O'Dell, C. S., \& ARNDT, D. R. (1981). Orientation in cognitive maps. Cognitive Psychology, 13, 149-206.

Huttenlocher, J., \& Presson, C. C. (1973). Mental rotation and the perspective problem. Cognitive Psychology, 4, 277-299.

Huttenlocher, J., \& Presson, C. C. (1979). The coding and transformation of spatial information. Cognitive Psychology, 11, 375-394.

KLATZKY, R. L. (1998). Allocentric and egocentric spatial representations: Definitions, distinctions, and interconnections. In C. Freksa, C. Habel, $\&$ K. F. Wender (Eds.), Spatial cognition: An interdisciplinaryapproach to representation and processing of spatial knowledge (Lecture Notes in Artificial Intelligence 1404, pp. 1-17). Berlin: Springer-Verlag.

Kornblum, S., HasbroucQ, T., \& OSMAn, A. (1990). Dimensional overlap: Cognitive basis for stimulus-response compatibility-a model and taxonomy. Psychological Review, 97, 253-270.

LeVine, M., Jankovic, I. N., \& PaliJ, M. (1982). Principles of spatial problem solving. Journal of Experimental Psychology: General, 111, $157-175$.
Liben, L. S., \& Downs, R. M. (1993). Understanding person-spacemap relations: Cartographic and developmental perspectives. Developmental Psychology, 29, 739-752.

LoGAN, G. D. (1995). Linguistic and conceptual control of visual spatial attention. Cognitive Psychology, 28, 103-174.

PRESSON, C. C. (1982). Strategies in spatial reasoning. Journal of Experimental Psychology: Learning, Memory, \& Cognition, 8, 243-251.

Presson, C. C., \& HAZElrigG, M. D. (1984). Building spatial representations through primary and secondary learning. Journal of Experimental Psychology: Learning, Memory, \& Cognition, 10, 516-528.

Presson, C. C., \& Montello, D. R. (1994). Updating after rotational and translational body movements: Coordinate structure of perspective space. Perception, 23, 1447-1455.

RIESER, J. J., GARING, A. E., \& Young, M. F. (1994). Imagery, action, and young children's spatial orientation: It's not being there that counts, it's what one has in mind. Child Development, 65, 1262-1278.

Roskos-Ewoldsen, B., McNamara, T. P., Shelton, A. L., \& CARr, W. (1998). Mental representations of large and small spatial layouts are orientation dependent. Journal of Experimental Psychology: Learning, Memory, \& Cognition, 24, 215-226.

SHE PARD, R. N., \& HuRWITZ, S. (1984). Upward direction, mental rotation, and discrimination of left and right turns in maps. Cognition, 18, 161-193.

Shepard, R. N., \& Metzler, J. (1972). Mental rotation of threedimensional objects. Science, 171, 701-703.

SHOLL, M. J. (1995). The representation and retrieval of map and environment knowledge. Geographical Systems, 2, 177-195.

Wraga, M., Creem, S. H., \& ProffitT, D. R. (1999). The influence of spatial reference frames on imagined object- and viewer rotations. Acta Psychologica, 102, 247-264.

(Manuscript received April 10, 2001; revision accepted for publication August 23, 2002.) 